Brit. J. industr. Med., 1963, 20, 324.

\title{
BLOOD GROUPS OF MINERS WITH COAL-WORKERS' PNEUMOCONIOSIS AND BRONCHITIS
}

\author{
BY \\ I. T. T. HIGGINS, P. D. OLDHAM, G. S. KILPATRICK, \\ R. J. DRUMMOND, and B. BEVAN \\ From the Medical Research Council's Epidemiological and Pneumoconiosis Research Units, \\ the Welsh National School of Medicine, and the Welsh Regional Transfusion Laboratory, Cardiff
}

(RECEIVED FOR PUBLICATION FEBRUARY 9, 1963)

An unexpected finding in a survey of a random sample of men from the Rhondda Fach valley in South Wales suggested an association between blood group A and coal-workers' pneumoconiosis. A further and larger sample of 1,250 miners and ex-miners from the same area, covering the same age range of 35 to 64 years, was chosen at random from the population in order to verify this finding. Each man was radiographed and questioned on his history and symptoms of chronic respiratory disease, and samples of blood and of saliva were obtained. The chest radiographs were classified according to the 1953 International Classification of Pneumoconiosis, and ABO and Rhesus blood groups and the secretor status were determined.

No convincing association between $\mathrm{ABO}$ or Rhesus blood groups or secretor status and either pneumoconiosis or respiratory symptoms associated with bronchitis was found. If an association between the blood groups and pneumoconiosis in fact existed, it would manifest itself as an effect on the response to exposure to dust. Future investigations should therefore be concerned with variations in the dose-response relation rather than with variations in the prevalence of pneumoconiosis.

During the analysis of the results of a field survey in 1958 in the Rhondda Fach, an excess of blood group $\mathrm{A}$ and a corresponding deficiency of blood group $\mathrm{O}$ were found among miners and ex-miners with pneumoconiosis (Table 1). The random sample of 600 persons consisted of equal numbers of miners and ex-miners and of non-miners divided into three decennial groups in the age range 35 to 64 years (Higgins and Cochrane, 1961; Kilpatrick and Hardisty, 1961). The only publication on blood group frequency in pneumoconiosis is that of Kuroda (referred to by Winkler, 1943), who found blood group B predominant in Japanese miners with silicosis.

This paper describes our studies in 1961 of a larger sample of miners and ex-miners in relation to pneumoconiosis and to bronchitis.

\section{Methods}

A sample of 1,250 miners and ex-miners was drawn from the population of the Rhondda Fach (defined by private census in the summer of 1958) by random numbers.
Details of the samples, the largest that could be studied in the time available, are given in Table 2.

All subjects were seen by appointment and samples of blood and saliva were obtained. A questionnaire similar to that approved in 1960 by the Medical Research Council's Bronchitis Committee was completed. "Chronic bronchitis" was defined as a persistent cough with sputum for some part of the day for at least three months together with one or more "chest illnesses". "Chest illness" was defined as one sufficiently severe to keep a man off work for a week or more with cough and sputum or an increase in these symptoms if they were usually present. The grades of breathlessness were those advocated by Fletcher (1952).

A $14 \times 14$ in. $(35.56 \times 35.56 \mathrm{~cm}$.) postero-anterior radiograph was taken of the chest and classified according to the International Classification (I.L.O., 1953). To avoid variation between observers, all questionnaires were completed, and all chest films were read by the same observer.

Blood grouping was performed using clotted blood samples; these were tested the day after collection. The methods used were those described in the Medical Research Council's Memorandum on ABO and $\mathrm{Rh}$ grouping (1958) and by Drummond (1961). 
TABLE 1

BLOOD GROUPS A, B, AND O, ACCORDING TO AGE, OCCUPATION, AND $X$-RAY CATEGORY OF PNEUMOCONIOSIS: RANDOM SAMPLE OF NON-MINERS, MINERS, AND EX-MINERS SEEN IN 1958

\begin{tabular}{|c|c|c|c|c|c|c|c|}
\hline \multirow{2}{*}{$\begin{array}{l}\text { Age } \\
\text { Group }\end{array}$} & \multirow{2}{*}{ Occupation } & \multirow{2}{*}{$X$-ray Category } & \multicolumn{5}{|c|}{ Blood Groups } \\
\hline & & & $\mathbf{O}$ & $\mathbf{A}$ & B & AB & Total \\
\hline $35-44$ & $\begin{array}{l}\text { Non-miners } \\
\text { Miners and ex-miners }\end{array}$ & $\begin{array}{c}\overline{\bar{O}} \\
\text { Simple pneumoconiosis } \\
\text { P.M.F. } \\
\text { Total: Miners and ex-miners }\end{array}$ & $\begin{array}{l}42(47 \cdot 7)^{*} \\
16(44 \cdot 4) \\
15(35 \cdot 7) \\
3(37 \cdot 5) \\
34(39 \cdot 5)\end{array}$ & $\begin{array}{r}32(36 \cdot 4) \\
15(41 \cdot 7) \\
23(54 \cdot 8) \\
3(37 \cdot 5) \\
41(47 \cdot 7)\end{array}$ & $\begin{aligned} & 10(11 \cdot 4) \\
& 4(11 \cdot 1) \\
& 3(7 \cdot 1) \\
& 2(25 \cdot 0) \\
& 9(10 \cdot 5)\end{aligned}$ & $\begin{array}{l}4(4 \cdot 5) \\
1(2 \cdot 8) \\
1(2 \cdot 4) \\
0(0 \cdot 0) \\
2(2 \cdot 3)\end{array}$ & $\begin{array}{r}88(100 \cdot 0) \\
36(100 \cdot 0) \\
42(100 \cdot 0) \\
8(100 \cdot 0) \\
86(100 \cdot 0)\end{array}$ \\
\hline $45-54$ & $\begin{array}{l}\text { Non-miners } \\
\text { Miners and ex-miners }\end{array}$ & $\begin{array}{c}\overline{\bar{O}} \\
\text { Simple pneumoconiosis } \\
\text { P.M.F. } \\
\text { Total: Miners and ex-miners }\end{array}$ & $\begin{array}{l}37(42 \cdot 0) \\
16(59 \cdot 3) \\
15(36 \cdot 6) \\
5(18 \cdot 5) \\
36(37 \cdot 9)\end{array}$ & $\begin{array}{l}42(47.7) \\
10(37.0) \\
22(53 \cdot 7) \\
14(51 \cdot 9) \\
46(48.4)\end{array}$ & $\begin{array}{c}5(5 \cdot 7) \\
1(3 \cdot 7) \\
3(7 \cdot 3) \\
6(22 \cdot 2) \\
10(10 \cdot 5)\end{array}$ & $\begin{array}{l}4(4 \cdot 5) \\
0(0 \cdot 0) \\
1(2 \cdot 4) \\
2(7 \cdot 4) \\
3(3 \cdot 2)\end{array}$ & $\begin{array}{l}88(99 \cdot 9) \\
27(100 \cdot 0) \\
41(100 \cdot 0) \\
27(100 \cdot 0) \\
95(100 \cdot 0)\end{array}$ \\
\hline $55-64$ & $\begin{array}{l}\text { Non-miners } \\
\text { Miners and ex-miners }\end{array}$ & $\begin{array}{c}\overline{\mathbf{O}} \\
\text { Simple pneumoconiosis } \\
\text { P.M.F. } \\
\text { Total: Miners and ex-miners }\end{array}$ & $\begin{array}{l}47(54 \cdot 7) \\
11(44 \cdot 0) \\
15(37 \cdot 5) \\
9(30 \cdot 0) \\
35(36 \cdot 8)\end{array}$ & $\begin{array}{l}28(32 \cdot 6) \\
11(44 \cdot 0) \\
19(47 \cdot 5) \\
17(56 \cdot 7) \\
47(49 \cdot 5)\end{array}$ & $\begin{array}{l}4(4 \cdot 7) \\
1(4 \cdot 0) \\
4(10 \cdot 0) \\
3(10 \cdot 0) \\
8(8 \cdot 4)\end{array}$ & $\begin{array}{l}7(8 \cdot 1) \\
2(8 \cdot 0) \\
2(5 \cdot 0) \\
1(3 \cdot 3) \\
5(5 \cdot 3)\end{array}$ & $\begin{array}{l}86(100 \cdot 0) \\
25(100 \cdot 0) \\
40(100 \cdot 0) \\
30(100 \cdot 0) \\
95(100 \cdot 0)\end{array}$ \\
\hline All ages & $\begin{array}{l}\text { Non-miners } \\
\text { Miners and ex-miners }\end{array}$ & $\begin{array}{c}\bar{O} \\
\text { Simple pneumoconiosis } \\
\text { Total: Miners and ex-miners }\end{array}$ & $\begin{array}{r}126(48 \cdot 1) \\
43(48 \cdot 9) \\
45(36 \cdot 6) \\
17(26 \cdot 2) \\
105(38 \cdot 0)\end{array}$ & \begin{tabular}{|r|}
$102(38 \cdot 9)$ \\
$36(40 \cdot 9)$ \\
$64(52 \cdot 0)$ \\
$34(52 \cdot 3)$ \\
$134(48 \cdot 6)$
\end{tabular} & $\begin{array}{l}19(7 \cdot 3) \\
6(6 \cdot 8) \\
10(8 \cdot 1) \\
11(16 \cdot 9) \\
27(9 \cdot 8)\end{array}$ & $\begin{array}{r}15(5 \cdot 7) \\
3(3 \cdot 4) \\
4(3 \cdot 2) \\
3(4 \cdot 6) \\
10(3 \cdot 6)\end{array}$ & $\begin{array}{r}262(100 \cdot 0) \\
88(100 \cdot 0) \\
123(99 \cdot 9) \\
65(100 \cdot 0) \\
276(100 \cdot 0)\end{array}$ \\
\hline
\end{tabular}

*Percentages in parentheses.

TABLE 2

RANDOM SAMPLES OF MINERS AND EX-MINERS STRATIFIED BY AGE

\begin{tabular}{|c|c|c|c|c|c|c|c|c|}
\hline Age Group & $\begin{array}{l}\text { No. in } \\
\text { Population } \\
\text { at Census* }\end{array}$ & No. Sampled & Excluded & Left Area & Dead & Refused & Examined & $\begin{array}{c}\text { No. in } \\
\text { Group after } \\
\text { Correcting Ages }\end{array}$ \\
\hline $\begin{array}{l}35-44 \\
45-54 \\
55-64\end{array}$ & $\begin{array}{l}1,137 \\
1,263 \\
1,168\end{array}$ & $\begin{array}{l}500(100 \cdot 0) \dagger \\
250(100 \cdot 0) \\
500(100 \cdot 0)\end{array}$ & $\begin{array}{l}5(1 \cdot 0) \\
1(0 \cdot 4) \\
2(0 \cdot 4)\end{array}$ & $\begin{array}{r}27(5.4) \\
9(3.6) \\
8(1.6)\end{array}$ & $\begin{array}{r}1(0 \cdot 2) \\
10(4 \cdot 0) \\
39(7 \cdot 8)\end{array}$ & $\begin{array}{l}27(5 \cdot 4) \\
23(9 \cdot 2) \\
57(7 \cdot 4)\end{array}$ & $\begin{array}{l}440(88 \cdot 0) \\
207(82 \cdot 8) \\
414(82 \cdot 8)\end{array}$ & $\begin{array}{l}440 \\
210 \\
411\end{array}$ \\
\hline Total & 3,568 & $1,250(100 \cdot 0)$ & $8(0.6)$ & $44(3 \cdot 5)$ & $50(4 \cdot 0)$ & $87(7 \cdot 0)$ & $1,061(84 \cdot 9)$ & 1,061 \\
\hline
\end{tabular}

*Date of census, June 1, 1958.

+Percentages are given in parentheses.

ABO Grouping.-Cells were tested with A, B, and O grouping sera. Sera were tested against $A$ and $B$ cells and for irregular antibodies, e.g. anti-Rh agglutinins. Tests were done to ensure that weakly reacting $A$ agglutinogen $\left(A_{3}\right.$, etc.) in group $\mathrm{A}$ or $\mathrm{AB}$ bloods did not escape detection. In bloods initially typed as group B, the cells were further tested with group $O$ serum having strong anti-A and which had been absorbed of its anti-B agglutinin. The sera of bloods typed initially as $B$ were also tested with $A_{1}$ and $A_{2}$ cells.

Rh Typing.-Cells were tested with two anti-D sera and those giving negative reactions were further tested with anti-C, D, and E sera. The $D^{\mathrm{u}}$ antigen was tested for by the indirect anti-globulin test after treatment of the cells with potent anti-D serum known to react with the $D^{u}$ antigen. Bloods classified as D-negative were those giving negative reactions with anti-D and anti- $D^{u}$ sera. Dnegative bloods comprise those which lack the $\mathrm{C}$ and $\mathrm{E}$ antigens or which contain one or other, or both, of these antigens.

Saliva Tests. - All salivas were tested independently by two observers for $A, B$, and $H$ substances. Anti-H extracted from seeds of Ulex europaea was used. One observer used a single tube technique with suitably diluted antisera. The other observer titrated the salivas. Any discrepancies between the results obtained were checked by repeating the tests if necessary on fresh specimens of saliva.

\section{Results}

Coal-workers' Pneumoconiosis.-Table 3 shows the prevalence of the various categories of pneumoconiosis in the sample. The proportion of men with pneumoconiosis was lower in this sample than in the previous one (Higgins and Cochrane, 1961). It seems probable that this is due to observer variation in film classification because in the present study all the radiographs were read by I.T.T.H. whereas in the previous sample all the radiographs were read by Professor A. L. Cochrane. At that time we commented that we had expected to find roughly equal numbers of miners and ex-miners with and without pneumoconiosis, whereas we had in fact found a deficiency of men with normal radiographs. The present findings are more in line with our earlier expectations. 
TABLE 3

PREVALENCE OF PNEUMOCONIOSIS IN THE SAMPLE

\begin{tabular}{|c|c|c|c|c|}
\hline \multirow{2}{*}{ Age Group } & \multicolumn{4}{|c|}{ Radiological Category } \\
\hline & No Pneumoconiosis & Simple Pneumoconiosis & P.M.F. & Total \\
\hline $\begin{array}{l}35-44 \\
45-54 \\
55-64\end{array}$ & $\begin{array}{l}259(58.9) * \\
103(49.0) \\
178(43.3)\end{array}$ & $\begin{array}{r}144(32 \cdot 7) \\
56(26 \cdot 7) \\
132(32 \cdot 1)\end{array}$ & $\begin{array}{c}37(8 \cdot 4) \\
51(24 \cdot 3) \\
101(24 \cdot 6)\end{array}$ & $\begin{array}{l}440(100 \cdot 0) \\
210(100 \cdot 0) \\
411(100 \cdot 0)\end{array}$ \\
\hline Total & $540(50 \cdot 9)$ & $332(31 \cdot 3)$ & $189(17 \cdot 8)$ & $1,061(100 \cdot 0)$ \\
\hline
\end{tabular}

*Percentages are given in parentheses

TABLE 4

A, B, O, AND RHESUS BLOOD GROUPS AND SECRETOR STATUS BY AGE AND RADIOLOGICAL CATEGORY OF PNEUMOCONIOSIS

\begin{tabular}{|c|c|c|c|c|c|c|c|c|}
\hline \multirow{2}{*}{$\begin{array}{c}\text { Age } \\
\text { Group }\end{array}$} & \multirow{2}{*}{ Radiological Category } & \multicolumn{5}{|c|}{ A, B, O Blood Group } & \multirow{2}{*}{$\begin{array}{l}\text { Rhesus } \\
\text { Group } \\
\text { Positive }\end{array}$} & \multirow{2}{*}{$\begin{array}{l}\text { Secretor } \\
\text { Status } \\
\text { Positive }\end{array}$} \\
\hline & & $\mathbf{O}$ & $\mathbf{A}$ & B & $\mathbf{A B}$ & Total & & \\
\hline \multirow[t]{2}{*}{$35-44$} & $\begin{array}{c}\text { O } \\
\text { Simple pneumoconiosis } \\
\text { P.M.F. }\end{array}$ & $\begin{array}{r}108(41 \cdot 7) \\
65(45 \cdot 1) \\
14(37 \cdot 8)\end{array}$ & $\begin{array}{r}118(45 \cdot 6) \\
57(39.6) \\
17(45.9)\end{array}$ & $\begin{array}{l}22(8 \cdot 5) \\
16(11 \cdot 1) \\
6(16 \cdot 2)\end{array}$ & $\begin{array}{r}11(4 \cdot 2) \\
6(4 \cdot 2) \\
0(0 \cdot 0)\end{array}$ & $\begin{array}{c}259(100.0) \\
144(100.0) \\
37(99.9)\end{array}$ & $\begin{array}{c}227(87 \cdot 6) \\
112(77 \cdot 8) \\
33(89 \cdot 2)\end{array}$ & $\begin{array}{c}194(74 \cdot 9) \\
96(66 \cdot 7) \\
26(72 \cdot 2)\end{array}$ \\
\hline & Total & $187(42 \cdot 5)$ & $192(43 \cdot 6)$ & $44(10 \cdot 0)$ & $17(3.9)$ & $440(100 \cdot 0)$ & $372(84 \cdot 5)$ & $316(72 \cdot 0)^{*}$ \\
\hline \multirow[t]{2}{*}{$45-54$} & $\begin{array}{c}\text { O } \\
\text { Simple pneumoconiosis } \\
\text { P.M.F. }\end{array}$ & $\begin{array}{l}53(51 \cdot 5) \\
23(41 \cdot 1) \\
19(37 \cdot 3)\end{array}$ & $\begin{array}{l}31(30 \cdot 1) \\
24(42 \cdot 9) \\
25(49 \cdot 0)\end{array}$ & $\begin{array}{c}14(13 \cdot 6) \\
6(10 \cdot 7) \\
5(9 \cdot 8)\end{array}$ & $\begin{array}{l}5(4 \cdot 9) \\
3(5 \cdot 4) \\
2(3 \cdot 9)\end{array}$ & $\begin{array}{r}103(100 \cdot 1) \\
56(100 \cdot 1) \\
51(100 \cdot 0)\end{array}$ & $\begin{array}{l}81(78 \cdot 6) \\
48(85 \cdot 7) \\
49(96 \cdot 1)\end{array}$ & $\begin{array}{l}78(75 \cdot 7) \\
40(71 \cdot 4) \\
40(78 \cdot 4)\end{array}$ \\
\hline & Total & $95(45 \cdot 2)$ & $80(38 \cdot 1)$ & $25(11 \cdot 9)$ & $10(4 \cdot 8)$ & $210(100 \cdot 0)$ & $178(84 \cdot 8)$ & $158(75 \cdot 2)$ \\
\hline \multirow[t]{2}{*}{$55-64$} & $\begin{array}{c}\text { O } \\
\text { Simple pneumoconiosis } \\
\text { P.M.F. }\end{array}$ & $\begin{array}{l}79(44 \cdot 4) \\
50(37 \cdot 9) \\
49(48 \cdot 5)\end{array}$ & $\begin{array}{l}72(40 \cdot 4) \\
61(46 \cdot 2) \\
39(38 \cdot 6)\end{array}$ & $\begin{array}{c}22(12 \cdot 4) \\
14(10 \cdot 6) \\
8(7 \cdot 9)\end{array}$ & $\begin{array}{l}5(2 \cdot 8) \\
7(5 \cdot 3) \\
5(5 \cdot 0)\end{array}$ & $\begin{array}{l}178(100 \cdot 0) \\
132(100 \cdot 0) \\
101(100 \cdot 0)\end{array}$ & $\begin{array}{r}142(79 \cdot 8) \\
106(80 \cdot 3) \\
82(81 \cdot 2)\end{array}$ & $\begin{array}{c}134(75 \cdot 3) \\
98(74 \cdot 8) \\
71(70 \cdot 3)\end{array}$ \\
\hline & Total & $178(43 \cdot 3)$ & $172(41 \cdot 8)$ & $44(10 \cdot 7)$ & $17(4 \cdot 1)$ & $411(99.9)$ & $330(80 \cdot 3)$ & $303(73.9)^{*}$ \\
\hline \multirow[t]{2}{*}{ Total } & $\begin{array}{c}\text { O } \\
\text { Simple pneumoconiosis } \\
\text { P.M.F. }\end{array}$ & $\begin{array}{r}240(44.4) \\
138(41.6) \\
82(43.4)\end{array}$ & $\begin{array}{r}221(40 \cdot 9) \\
142(42 \cdot 8) \\
81(42 \cdot 9)\end{array}$ & $\begin{array}{l}58(10 \cdot 7) \\
36(10 \cdot 8) \\
19(10 \cdot 1)\end{array}$ & $\begin{array}{r}21(3 \cdot 9) \\
16(4 \cdot 8) \\
7(3 \cdot 7)\end{array}$ & $\begin{array}{l}540(99 \cdot 9) \\
332(100 \cdot 0) \\
189(100 \cdot 1)\end{array}$ & $\begin{array}{l}450(83 \cdot 3) \\
266(80 \cdot 1) \\
164(86 \cdot 8)\end{array}$ & $\begin{array}{l}406(75 \cdot 2) \\
234(70 \cdot 7)^{*} \\
137(72 \cdot 9)^{*}\end{array}$ \\
\hline & Total & $460(43.4)$ & $444(41 \cdot 8)$ & $113(10 \cdot 7)$ & $44(4 \cdot 2)$ & $1061(100 \cdot 1)$ & $880(82 \cdot 9)$ & $777(73.4)^{*}$ \\
\hline
\end{tabular}

*In two cases samples of saliva were not obtained.

Table 4 shows the frequency of $\mathrm{ABO}$ and Rhesus blood groups according to age and radiological category. When all age groups are considered together, the frequencies of groups $\mathrm{O}$ and $\mathrm{A}$ are found to be 44.4 and $40.9 \%$ in the men without pneumoconiosis and 42.2 and $42.8 \%$ in those with pneumoconiosis. The differences between these percentages are quite insignificant. When individual age groups are considered separately there is again no suggestion of an increased frequency of blood group A among miners with pneumoconiosis. Only in the 45-54 group (the smallest) are there differences in the blood group frequency that are at all suggestive. Here there is a deficiency of group $\mathrm{A}$ and a corresponding excess of group $\mathrm{O}$ among the miners without pneumoconiosis, a result that is in accordance with the findings of the earlier survey. Indeed, the differences between the three age groups in this survey approach a significant level $\left(\chi^{2}=5.12\right.$ with two degrees of freedom, $0 \cdot 10>p>0.05)$.

The proportions of $R h$ positive subjects among the men with pneumoconiosis and the men without vary significantly between the three age groups. Among the men aged 35 to 44 the proportion of $R h$ positive subjects is somewhat lower when pneumoconiosis was diagnosed than when it was not; among those aged 45 to 54 the proportions are reversed, to an extent which in isolation appears significant $(0.02>p$ $>0.01$ ); among the men aged 55 to 64 there is no apparent difference. This variation between age groups is unlikely to be due to chance $\left(\chi^{2}=10 \cdot 18\right.$ for two degrees of freedom, $0.01>p>0.001$ ).

Respiratory Symptoms and Bronchitis.-Table 5 shows the prevalence of respiratory symptoms in the sample according to age and radiological category of pneumoconiosis. As age advances, symptoms in each category become more frequent. There is a clear relation in this sample between respiratory symptoms and the radiological category of pneumoconiosis. In each group the men with simple pneumoconiosis had a higher prevalence of symptoms than those without though the difference is sometimes small, and those with P.M.F. recorded an even higher prevalence. An 
TABLE 5

PREVALENCE OF RESPIRATORY SYMPTOMS AND “CHRONIC BRONCHITIS" ACCORDING TO AGE AND RADIOLOGICAL CATEGORY OF PNEUMOCONIOSIS

\begin{tabular}{|c|c|c|c|c|c|c|c|c|c|c|c|}
\hline \multirow[b]{2}{*}{$\begin{array}{c}\text { Age } \\
\text { Group }\end{array}$} & \multirow{2}{*}{$\begin{array}{c}\text { Radiological } \\
\text { Category of } \\
\text { Pneumoconiosis }\end{array}$} & \multirow[b]{2}{*}{ No. ${ }^{1}$} & \multirow[b]{2}{*}{ Cough } & \multirow[b]{2}{*}{ Sputum } & \multirow{2}{*}{$\begin{array}{c}\text { Cough } \\
\text { and } \\
\text { Sputum }\end{array}$} & \multicolumn{3}{|c|}{ Chest Illness ${ }^{2}$} & \multicolumn{2}{|c|}{ Breathlessness $^{3}$} & \multirow{2}{*}{$\begin{array}{l}\text { "Chronic" } \\
\text { Bronchitis" }\end{array}$} \\
\hline & & & & & & Once & $\begin{array}{l}\text { More than } \\
\text { Once }\end{array}$ & Total & $\begin{array}{c}\text { Grades } 2 \\
\text { and Over }\end{array}$ & $\begin{array}{l}\text { Grades } 3 \\
\text { and Over }\end{array}$ & \\
\hline \multirow[t]{3}{*}{$35-44$} & $\underset{\text { Simp }}{\mathbf{O}}$ & 259 & $(42 \cdot 1)$ & $106(40 \cdot 9)$ & $80(30.9)$ & $24(9 \cdot 3)$ & $18(6.9)$ & $42(16 \cdot 2)$ & $47(18 \cdot 1)$ & $21(8 \cdot 1)$ & $25(9 \cdot 7)$ \\
\hline & $\begin{array}{l}\text { pneumoconiosis } \\
\text { P.M.F. }\end{array}$ & $\begin{array}{r}144 \\
37\end{array}$ & $\begin{array}{l}72(50 \cdot 0) \\
22(61 \cdot 1)\end{array}$ & $\begin{array}{l}71(49 \cdot 3) \\
24(66 \cdot 7)\end{array}$ & $\begin{array}{l}57(39 \cdot 6) \\
21(58 \cdot 3)\end{array}$ & $\begin{array}{r}14(9 \cdot 7) \\
3(8 \cdot 3)\end{array}$ & $\begin{array}{r}15(10.4) \\
8(22 \cdot 2)\end{array}$ & $\begin{array}{l}29(20 \cdot 1) \\
11(30 \cdot 5)\end{array}$ & $\begin{array}{l}54(37 \cdot 5) \\
15(41 \cdot 7)\end{array}$ & $\begin{array}{r}16(11 \cdot 1) \\
7(19 \cdot 4)\end{array}$ & $\begin{array}{r}18(12 \cdot 5) \\
8(22 \cdot 2)\end{array}$ \\
\hline & Total & 440 & $203(46 \cdot 2)$ & $201(45 \cdot 8)$ & $158(36 \cdot 0)$ & $41(9 \cdot 3)$ & $41(9 \cdot 3)$ & $82(18 \cdot 7)$ & $116(26 \cdot 4)$ & $44(10 \cdot 0)$ & $51(11 \cdot 6)$ \\
\hline \multirow[t]{3}{*}{$45-54$} & & 103 & $50(48 \cdot 5)$ & $53(51 \cdot 5)$ & $42(40 \cdot 8)$ & $8(7 \cdot 8)$ & $17(16 \cdot 5)$ & $25(24 \cdot 3)$ & $37(35.9)$ & $12(11 \cdot 7)$ & $16(15 \cdot 5)$ \\
\hline & $\begin{array}{l}\text { pneumoconiosis } \\
\text { P.M.F. }\end{array}$ & $\begin{array}{l}56 \\
51\end{array}$ & $\begin{array}{l}37(66 \cdot 1) \\
35(68 \cdot 6)\end{array}$ & $\begin{array}{l}36(64 \cdot 3) \\
34(66 \cdot 7)\end{array}$ & $\begin{array}{l}28(50.0) \\
29(56.9)\end{array}$ & $\begin{array}{c}5(8 \cdot 9) \\
11(21 \cdot 6)\end{array}$ & $\begin{array}{l}9(16 \cdot 1) \\
9(17 \cdot 6)\end{array}$ & $\begin{array}{l}14(25 \cdot 0) \\
20(39 \cdot 2)\end{array}$ & $\begin{array}{l}29(51 \cdot 8) \\
33(64 \cdot 7)\end{array}$ & $\begin{array}{l}10(17 \cdot 9) \\
12(23 \cdot 5)\end{array}$ & $\begin{array}{l}13(23 \cdot 2) \\
16(31 \cdot 4)\end{array}$ \\
\hline & Total & 210 & $122(58 \cdot 1)$ & $123(58 \cdot 6)$ & $99(47 \cdot 1)$ & $24(11 \cdot 4)$ & $35(16 \cdot 7)$ & $59(28 \cdot 1)$ & $99(47 \cdot 1)$ & $34(16 \cdot 2)$ & $45(21 \cdot 4)$ \\
\hline \multirow[t]{3}{*}{$55-64$} & & 178 & $107(60 \cdot 1)$ & $93(52 \cdot 2)$ & $79(44 \cdot 4)$ & $12(6 \cdot 9)$ & $32(18 \cdot 3)$ & $44(25 \cdot 1)$ & $78(43 \cdot 8)$ & $46(25 \cdot 8)$ & $32(18 \cdot 2)$ \\
\hline & $\begin{array}{l}\text { pneumoconiosis } \\
\text { P.M.F. }\end{array}$ & $\begin{array}{l}132 \\
101\end{array}$ & $\begin{array}{l}78(60.0) \\
70(70 \cdot 0)\end{array}$ & $\begin{array}{l}75(57.7) \\
69(69 \cdot 0)\end{array}$ & $\begin{array}{l}63(48 \cdot 5) \\
61(61 \cdot 0)\end{array}$ & $\begin{array}{l}10(7 \cdot 7) \\
14(14 \cdot 1)\end{array}$ & $\begin{array}{l}26(20 \cdot 0) \\
27(27 \cdot 3)\end{array}$ & $\begin{array}{l}36(27 \cdot 7) \\
41(41 \cdot 4)\end{array}$ & $\begin{array}{l}71(54 \cdot 6) \\
68(68 \cdot 7)\end{array}$ & $\begin{array}{l}45(34 \cdot 6) \\
41(41 \cdot 4)\end{array}$ & $\begin{array}{l}29(22 \cdot 3) \\
29(29 \cdot 3)\end{array}$ \\
\hline & Total & 411 & $255(62 \cdot 5)$ & $237(58 \cdot 1)$ & $203(49 \cdot 8)$ & $36(8.9)$ & $85(21 \cdot 0)$ & $121(30 \cdot 0)$ & $217(53 \cdot 3)$ & $132(32 \cdot 4)$ & $90(22 \cdot 2)$ \\
\hline
\end{tabular}

${ }^{1}$ In one man aged 35-44 with P.M.F., and two men with simple pneumoconiosis and one man with P.M.F. aged 55-64, respiratory symptoms were not recorded.

In three men category $O$ and one man with P.M.F. aged 55-64 chest illness was not recorded.

${ }^{2}$ In one man with P.M.F. aged 55-64 breathlessness was not recorded.

"In two men category $O$ and one man with P.M.F. aged 55-64 "chronic bronchitis" was not recorded.

TABLE 6

RESPIRATORY SYMPTOMS AND "CHRONIC BRONCHITIS" ACCORDING TO AGE AND BLOOD GROUP

\begin{tabular}{|c|c|c|c|c|c|c|c|c|c|c|c|}
\hline \multirow{2}{*}{$\underset{\text { Group }}{\text { Age }}$} & \multirow{2}{*}{$\begin{array}{l}\text { Blood } \\
\text { Group }\end{array}$} & \multirow[b]{2}{*}{ No. } & \multirow{2}{*}{ Cough } & \multirow{2}{*}{ Sputum } & \multirow{2}{*}{$\begin{array}{l}\text { Cough } \\
\text { and } \\
\text { Sputum }\end{array}$} & \multicolumn{3}{|c|}{ Chest Illness } & \multicolumn{2}{|c|}{ Breathlessness } & \multirow{2}{*}{$\begin{array}{l}\text { "Chronic } \\
\text { Bronchitis" }\end{array}$} \\
\hline & & & & & & Once & $\begin{array}{c}\text { More than } \\
\text { Once }\end{array}$ & Total & $\begin{array}{l}\text { Grades } 2 \\
\text { and over }\end{array}$ & $\begin{array}{l}\text { Grades } 3 \\
\text { and over }\end{array}$ & \\
\hline \multirow[t]{2}{*}{$35-44$} & $\begin{array}{c}\mathbf{O} \\
\mathbf{A} \\
\mathbf{B} \\
\mathbf{A B}\end{array}$ & $\begin{array}{r}187 \\
192 \\
44 \\
17\end{array}$ & $\begin{array}{r}86(46.0) \\
84(44.0) \\
24(54 \cdot 5) \\
9(52.9)\end{array}$ & $\begin{array}{r}91(48 \cdot 7) \\
82(42 \cdot 9) \\
20(45 \cdot 5) \\
8(47 \cdot 1)\end{array}$ & $\begin{array}{l}71(38 \cdot 0) \\
61(31 \cdot 9) \\
19(43 \cdot 2) \\
7(41 \cdot 2)\end{array}$ & $\begin{array}{c}17(9 \cdot 1) \\
15(7 \cdot 9) \\
7(15 \cdot 9) \\
2(11 \cdot 8)\end{array}$ & $\begin{array}{c}18(9 \cdot 6) \\
15(7.9) \\
6(13 \cdot 6) \\
2(11 \cdot 8)\end{array}$ & $\begin{array}{r}35(18.7) \\
30(15.7) \\
13(29.5) \\
4(23.5)\end{array}$ & $\begin{array}{r}53(28 \cdot 3) \\
47(24.6) \\
24(54.5) \\
3(11.8)\end{array}$ & $\begin{array}{c}22(11 \cdot 8) \\
18(9 \cdot 4) \\
3(6 \cdot 8) \\
1(5 \cdot 9)\end{array}$ & $\begin{array}{c}23(12 \cdot 3) \\
19(9 \cdot 9) \\
6(13 \cdot 6) \\
3(17 \cdot 6)\end{array}$ \\
\hline & Total & 440 & $203(46 \cdot 2)$ & $201(45 \cdot 8)$ & $158(36.0)$ & $41(9 \cdot 3)$ & $41(9 \cdot 3)$ & $82(18 \cdot 7)$ & $116(26 \cdot 4)$ & $44(10 \cdot 0)$ & $51(11 \cdot 6)$ \\
\hline \multirow[t]{2}{*}{$45-54$} & $\begin{array}{c}\mathbf{O} \\
\mathbf{A} \\
\mathbf{B} \\
\mathbf{A B}\end{array}$ & $\begin{array}{l}95 \\
80 \\
25 \\
10\end{array}$ & $\begin{array}{r}53(55 \cdot 8) \\
46(57 \cdot 5) \\
17(68.0) \\
6(60.0)\end{array}$ & $\begin{array}{r}61(63 \cdot 1) \\
43(53 \cdot 8) \\
16(64 \cdot 0) \\
3(30 \cdot 0)\end{array}$ & $\begin{array}{r}44(46 \cdot 3) \\
37(46 \cdot 3) \\
15(60 \cdot 0) \\
3(30 \cdot 0)\end{array}$ & $\begin{array}{c}9(9 \cdot 5) \\
12(15.0) \\
3(12.0) \\
0(0.0)\end{array}$ & $\begin{array}{c}22(23 \cdot 2) \\
9(11 \cdot 3) \\
3(12 \cdot 0) \\
1(10 \cdot 0)\end{array}$ & $\begin{array}{r}31(32.6) \\
21(26 \cdot 3) \\
6(24 \cdot 0) \\
1(10.0)\end{array}$ & $\begin{array}{l}47(49 \cdot 5) \\
37(46 \cdot 3) \\
12(48 \cdot 0) \\
3(30 \cdot 0)\end{array}$ & $\begin{array}{c}18(18.9) \\
14(17.5) \\
1(4.0) \\
1(10.0)\end{array}$ & $\begin{array}{r}24(25 \cdot 3) \\
16(20 \cdot 0) \\
4(16 \cdot 0) \\
1(10 \cdot 0)\end{array}$ \\
\hline & Total & 210 & $122(58 \cdot 1)$ & $123(58 \cdot 6)$ & $99(47 \cdot 1)$ & $24(11 \cdot 4)$ & $35(16 \cdot 7)$ & $59(28 \cdot 1)$ & $99(47 \cdot 1)$ & $34(16 \cdot 2)$ & $45(21 \cdot 4)$ \\
\hline $55-64$ & $\begin{array}{c}\mathbf{O} \\
\mathbf{A} \\
\mathbf{B} \\
\mathbf{A B}\end{array}$ & $\begin{array}{r}178 \\
172 \\
44 \\
17\end{array}$ & $\begin{array}{r}110(62 \cdot 1) \\
109(63 \cdot 7) \\
27(62.8) \\
9(52.9)\end{array}$ & $\begin{array}{r}101(57 \cdot 1) \\
104(60 \cdot 8) \\
21(48 \cdot 8) \\
11(64 \cdot 7)\end{array}$ & $\begin{array}{l}88(49 \cdot 7) \\
87(50 \cdot 9) \\
20(46 \cdot 5) \\
8(47 \cdot 1)\end{array}$ & $\begin{array}{c}13(7 \cdot 4) \\
17(10 \cdot 1) \\
4(9 \cdot 3) \\
2(11 \cdot 8)\end{array}$ & $\begin{array}{c}33(18.9) \\
37(21.9) \\
12(27.9) \\
3(17.6)\end{array}$ & $\begin{array}{r}46(26 \cdot 3) \\
54(32 \cdot 0) \\
16(37 \cdot 2) \\
5(29 \cdot 4)\end{array}$ & $\begin{array}{l}90(50.8) \\
95(55.6) \\
23(54.8) \\
9(52.9)\end{array}$ & $\begin{array}{r}52(29 \cdot 4) \\
64(37 \cdot 4) \\
11(26 \cdot 2) \\
5(29 \cdot 4)\end{array}$ & $\begin{array}{r}29(16 \cdot 5) \\
44(26 \cdot 0) \\
13(30 \cdot 2) \\
4(23 \cdot 5)\end{array}$ \\
\hline \multirow[t]{2}{*}{$35-64$} & $\begin{array}{c}\mathbf{O} \\
\mathbf{A} \\
\mathbf{B} \\
\mathbf{A B}\end{array}$ & $\begin{array}{r}460 \\
444 \\
113 \\
44\end{array}$ & $\begin{array}{r}249(54 \cdot 2) \\
239(54 \cdot 1) \\
68(60 \cdot 7) \\
24(54 \cdot 5)\end{array}$ & $\begin{array}{r}253(55 \cdot 1) \\
229(51 \cdot 8) \\
57(50 \cdot 9) \\
22(50 \cdot 0)\end{array}$ & $\begin{array}{r}203(44 \cdot 2) \\
185(41 \cdot 9) \\
54(48 \cdot 2) \\
18(40 \cdot 9)\end{array}$ & $\begin{array}{c}39(8 \cdot 5) \\
44(10 \cdot 0) \\
14(12 \cdot 5) \\
4(9 \cdot 1)\end{array}$ & $\begin{array}{r}73(16.0) \\
61(13.9) \\
21(18.8) \\
6(13.6)\end{array}$ & $\begin{array}{r}112(24 \cdot 5) \\
105(23 \cdot 9) \\
35(31 \cdot 3) \\
10(22 \cdot 7)\end{array}$ & $\begin{array}{r}190(41 \cdot 4) \\
179(40 \cdot 5) \\
49(44 \cdot 1) \\
14(31 \cdot 8)\end{array}$ & $\begin{array}{r}92(20.0) \\
96(21.7) \\
15(13.5) \\
7(15.9)\end{array}$ & $\begin{array}{r}76(16 \cdot 6) \\
79(18 \cdot 0) \\
23(20 \cdot 5) \\
8(18 \cdot 2)\end{array}$ \\
\hline & Total & 1,061 & $580(54.9)$ & $561(53 \cdot 1)$ & $460(43 \cdot 5)$ & $101(9 \cdot 6)$ & $161(15 \cdot 3)$ & $262(24 \cdot 9)$ & $432(40.9)$ & $210(19.9)$ & $186(17 \cdot 6)$ \\
\hline
\end{tabular}

increasing prevalence of respiratory symptoms with increasing radiological category of pneumoconiosis was noted by Rogan, Ashford, Chapman, Duffield, Fay, and Rae (1961) when the findings in six British collieries surveyed by the National Coal Board were combined.

Table 6 shows the prevalence of respiratory symptoms and "chronic bronchitis" according to age and blood group. There is no evidence of any association between symptoms and blood group. This agrees with the observations of Lewis and Woods (1961), who studied the blood groups in six respiratory disorders in patients referred to the Brompton Hospital. They found no evidence of any association between blood group and cases diagnosed as bronchitis and emphysema. 
TABLE 7

PREVALENCE OF SYMPTOMS ACCORDING TO AGE AND SECRETOR STATUS

\begin{tabular}{|c|c|c|c|c|c|c|c|c|c|c|c|}
\hline \multirow{2}{*}{$\begin{array}{l}\text { Age } \\
\text { Group }\end{array}$} & \multirow{2}{*}{$\begin{array}{l}\text { Secretor } \\
\text { Status }\end{array}$} & \multirow[b]{2}{*}{ No. } & \multirow[b]{2}{*}{ Cough } & \multirow{2}{*}{ Sputum } & \multirow{2}{*}{$\begin{array}{l}\text { Cough } \\
\text { and } \\
\text { Sputum }\end{array}$} & \multicolumn{3}{|c|}{ Chest Illness } & \multicolumn{2}{|c|}{ Breathlessness } & \multirow{2}{*}{$\begin{array}{c}\text { "Chronic } \\
\text { Bronchitis", }\end{array}$} \\
\hline & & & & & & Once & $\begin{array}{c}\text { More than } \\
\text { Once }\end{array}$ & Total & $\begin{array}{l}\text { Grades } 2 \\
\text { and Over }\end{array}$ & $\begin{array}{l}\text { Grades } 3 \\
\text { and Over }\end{array}$ & \\
\hline $35-44$ & $\begin{array}{l}\text { Positive } \\
\text { Negative }\end{array}$ & $\begin{array}{l}316 \\
123\end{array}$ & $\begin{array}{r}147(46 \cdot 5) \\
56(45 \cdot 5)\end{array}$ & $\begin{array}{r}147(46.5) \\
54(43.9)\end{array}$ & $\begin{array}{r}115(36.4) \\
43(35.0)\end{array}$ & $\begin{array}{l}30(9 \cdot 5) \\
11(8 \cdot 9)\end{array}$ & $\begin{array}{c}33(10 \cdot 4) \\
8(6 \cdot 5)\end{array}$ & $\begin{array}{l}63(19.9) \\
19(15.4)\end{array}$ & $\begin{array}{l}87(27 \cdot 5) \\
29(23 \cdot 6)\end{array}$ & $\begin{array}{l}34(10 \cdot 8) \\
10(8 \cdot 1)\end{array}$ & $\begin{array}{l}38(12.0) \\
13(10.6)\end{array}$ \\
\hline $45-54$ & $\begin{array}{l}\text { Positive } \\
\text { Negative }\end{array}$ & $\begin{array}{r}158 \\
52\end{array}$ & $\begin{array}{l}90(57 \cdot 0) \\
32(61 \cdot 5)\end{array}$ & $\begin{array}{l}93(58.9) \\
30(57.7)\end{array}$ & $\begin{array}{l}73(46 \cdot 2) \\
26(50 \cdot 0)\end{array}$ & $\begin{array}{c}21(13 \cdot 3) \\
3(5 \cdot 7)\end{array}$ & $\begin{array}{r}27(17 \cdot 1) \\
8(15 \cdot 4)\end{array}$ & $\begin{array}{l}48(30.4) \\
11(21.4)\end{array}$ & $\begin{array}{l}79(50 \cdot 0) \\
20(38 \cdot 5)\end{array}$ & $\begin{array}{r}25(15 \cdot 8) \\
9(17 \cdot 3)\end{array}$ & $\begin{array}{r}36(22 \cdot 8) \\
9(17 \cdot 3)\end{array}$ \\
\hline $55-64$ & $\begin{array}{l}\text { Positive } \\
\text { Negative }\end{array}$ & $\begin{array}{l}303 \\
107\end{array}$ & $\begin{array}{r}196(65 \cdot 1) \\
59(55 \cdot 1)\end{array}$ & $\begin{array}{r}177(58 \cdot 8) \\
60(56 \cdot 1)\end{array}$ & $\begin{array}{r}153(50.8) \\
50(46.7)\end{array}$ & $\begin{array}{r}27(9 \cdot 1) \\
9(8 \cdot 4)\end{array}$ & $\begin{array}{l}62(20 \cdot 9) \\
23(21 \cdot 5)\end{array}$ & $\begin{array}{l}89(30.0) \\
32(29.9)\end{array}$ & $\begin{array}{r}156(52 \cdot 0) \\
61(57 \cdot 0)\end{array}$ & $\begin{array}{l}91(30 \cdot 3) \\
41(38 \cdot 3)\end{array}$ & $\begin{array}{l}68(22 \cdot 8) \\
22(20 \cdot 6)\end{array}$ \\
\hline Total & $\begin{array}{l}\text { Positive } \\
\text { Negative }\end{array}$ & $\begin{array}{l}777 \\
282\end{array}$ & $\begin{array}{l}433(55 \cdot 9) \\
147(52 \cdot 1)\end{array}$ & $\begin{array}{l}417(53 \cdot 8) \\
144(51 \cdot 1)\end{array}$ & \begin{tabular}{|l}
$341(44 \cdot 0)$ \\
$119(42 \cdot 2)$
\end{tabular} & $\begin{array}{l}78(10 \cdot 1) \\
23(8 \cdot 2)\end{array}$ & $\begin{array}{r}122(15 \cdot 8) \\
39(13 \cdot 8)\end{array}$ & $\begin{array}{r}200(25.9) \\
62(22.0)\end{array}$ & \begin{tabular}{|l}
$322(41 \cdot 5)$ \\
$110(39 \cdot 0)$
\end{tabular} & $\begin{array}{r}150(19 \cdot 4) \\
60(21 \cdot 3)\end{array}$ & $\begin{array}{r}142(18 \cdot 4) \\
44(15 \cdot 6)\end{array}$ \\
\hline
\end{tabular}

TABLE 8

BLOOD GROUP FREQUENCIES ACCORDING TO RADIOLOGICAL CATEGORY IN THE 1958 AND 1961 SAMPLES

\begin{tabular}{|c|c|c|c|c|c|c|}
\hline \multirow{2}{*}{ Radiological Category } & \multirow{2}{*}{ Year } & \multicolumn{5}{|c|}{ Blood Group } \\
\hline & & $\mathbf{O}$ & $\mathbf{A}$ & B & $\mathbf{A B}$ & Total \\
\hline $\mathbf{O}$ & $\begin{array}{l}1958 \\
1961\end{array}$ & $\begin{array}{r}43(48.9) \\
240(44.4)\end{array}$ & $\begin{array}{r}36(40.9) \\
221(40.9)\end{array}$ & $\begin{array}{c}6(6 \cdot 8) \\
58(10 \cdot 7)\end{array}$ & $\begin{array}{r}3(3.4) \\
21(3.9)\end{array}$ & $\begin{array}{r}88(100 \cdot 0) \\
540(99 \cdot 9)\end{array}$ \\
\hline Pneumoconiosis & $\begin{array}{l}1958 \\
1961\end{array}$ & $\begin{array}{r}62(33 \cdot 0) \\
220(42 \cdot 2)\end{array}$ & $\begin{array}{r}98(52 \cdot 1) \\
223(42 \cdot 8)\end{array}$ & $\begin{array}{l}21(11 \cdot 2) \\
55(10 \cdot 6)\end{array}$ & $\begin{array}{r}7(3 \cdot 7) \\
23(4 \cdot 4)\end{array}$ & $\begin{array}{l}188(100 \cdot 0) \\
521(100 \cdot 0)\end{array}$ \\
\hline Total & $\begin{array}{l}1958 \\
1961\end{array}$ & $\begin{array}{l}105(38.0) \\
460(43.4)\end{array}$ & $\begin{array}{l}134(48 \cdot 6) \\
444(41 \cdot 8)\end{array}$ & $\begin{array}{c}27(9 \cdot 8) \\
113(10 \cdot 7)\end{array}$ & $\begin{array}{l}10(3 \cdot 6) \\
44(4 \cdot 1)\end{array}$ & $\begin{array}{r}276(100 \cdot 0) \\
1,061(100 \cdot 0)\end{array}$ \\
\hline
\end{tabular}

Table 7 indicates that secretor status also appears to be unimportant in this respect. The age standardized prevalence of respiratory symptoms is the same in both secretor-positive and secretor-negative groups.

\section{Discussion}

The impression of an association between blood groups $\mathrm{O}$ and $\mathrm{A}$ and pneumoconiosis suggested by the small sample drawn in 1958 has not been strongly supported by the larger sample, drawn from virtually the same population, in 1961. Table 8 brings the relevant results together. The excess of group $A$ in men with pneumoconiosis (and a corresponding deficiency of group $\mathbf{O}$ ) is still present but is extremely small, and the combined evidence from the two samples only attains the $10 \%$ level of significance. There is no convincing evidence of heterogeneity of the two samples ( $p \bumpeq 0.08$ ), so it appears that the play of chance may well have been responsible for the differences between them.

Associations between characteristics observed in samples in which many such characteristics have been recorded will appear, by chance, with a frequency larger than that suggested by a single test of significance. If it were known how many pairs of characteristics had been examined for association, the chance frequency could be calculated; in practice, the suggestion of Fraser Roberts (1955) that a significance level of $1 / 1,000$ should be demanded in blood group work before an association is accepted provides a reasonable safeguard.

When the hypothesis of a particular kind of association has been formed by inspection of sample data not specifically collected with this hypothesis in mind, it is well not to accept the result of a conventional test of significance but to explore the hypothesis by means of a new sample, as we have done in this case with the suggested association between pneumoconiosis and the $\mathrm{O} / \mathrm{A}$ ratio. In the new sample we have now found apparent evidence of heterogeneity in the relation of pneumoconiosis to $\mathrm{Rh}$ positivity. It is clear that further studies are necessary before this can be accepted.

It is of some interest to consider what form of association would be expected if there were a connexion between pneumoconiosis and the blood groups. The very extensive evidence that the prevalence of pneumoconiosis can be related to exposure to dust by some form of dose-response curve (Fay and Ashford, 1961) suggests that any effect of the blood group would be in modifying the shape of this curve, the speed of radiological response to dust inhalation, rather than in producing similar differences in prevalence among men of all ages, of widely differing dust exposures. Unless the blood group in some way determines the dust exposure of an 
individual, which seems unlikely, one would expect differences in prevalence to be small among young men, to increase with age (and thus dust exposure), remaining large or perhaps diminishing again among the oldest men. Alternatively, it may be the risk of massive fibrosis associated with a given degree of simple pneumoconiosis which depends on the blood group. It may therefore be better in future studies to attempt to distinguish the dose-response curves of groups of men of different blood groups rather than to proceed with further studies of prevalence unrelated to dust exposure.

We are grateful to Drs. N. Pearson and D. C. Morgan, of the National Coal Board, for urging us to analyse the blood groups from the first survey in relation to pneumoconiosis; to Dr. J. A. Fraser Roberts for advising us to repeat our observations on a larger sample and, together with Professor $\mathrm{H}$. Scarborough, for reading and criticizing the manuscript; to our colleagues at the Pneumoconiosis and Epidemiological Research Units for their help in carrying out the surveys.

\section{REFERENCES}

Drummond, R. J. (1961). Blood transfusion. In The Extra Pharmacopoeia (Martindale), Supplement, p. 93. Pharmaceutical Press, London.

Fay, J. W. J. and Ashford, J. R. (1961), Brit. J. industr. Med., 18, 175. Fletcher, C. M. (1952). Proc. roy. Soc. Med. 45, 577. Higgins, I. T. T., and Cochrane, A. L. (1961). Brit. J. industr. Med.,

Kilpatrick, G. S., and Hardisty, R. M. (1961). Brit. med. J., 1, 778

Medical Research Council (1958). Memorandum No.' 36. The determination of the ABO and Rh (D) blood groups for transfusion. H.M.S.O., London.

Roberts, J. A. Fraser (1955). Proc. roy. Soc. Med., 48, 143.

Rogan, J. M., Ashford, J. R., Chapman, P. J., Duffield, D. P., Fay, J. W. J., and Rae, S. (1961). Brit. med. J., 1, 1337.

Winkler, A. (1943). Beitr. Klin. Tuberk., 99, 130.

\section{THE JULY (1963) ISSUE}

The July (1963) issue contains the following papers:-

The Successful Prevention of Lead Poisoning in the Glazing of Earthenware in the North Staffordshire Potteries. A. MeIKLEJOHN

Dust and Collagen Content of Lungs of Coal-workers with Progressive Massive Fibrosis. G. NAGELSCHMIDT, D. Rivers, E. J. King, and W. TREVElla

Chromate Dermatitis from Paint. H. O. Engel and C. D. Calnan

A Cause of Chromate Dermatitis among Assemblers in an Automobile Factory. MurIEL L. Newhouse

Studies of Ventilatory Capacity and Histamine Response during Exposure to Isocyanate Vapour in Polyurethane Foam Manufacture. BRYAN GANDEVIA

An Account of the Founding of H.M. Inspectorate of Mines and the Work of the First Inspector Hugh Seymour Tremenheere. O. P. EDMONDS and E. L. EDMONDS

Silicosis in West Country Granite Workers. L. W. HALE and G. SHeErS

Pneumoconiosis in China-clay Workers. S. WARRAKI and Y. Herant

The Reliability of Repeated Auditory Threshold Determination. G. R. C. AtherLeY and I. DingwallFORDYCE

An Autopsy Survey of Bantu South African Coal-miners. C. B. Chatgidakis

Changes in Activity of Respiratory Enzymes in Lungs of Guinea-pigs Exposed to Silica Dust. T. A. KILROESMITH and MARIA G. BREYER

The Collection of Expired Volatile Substances. J. C. GAGE

Miscellanea

Byssinosis in Great Britain. A. MeIKLejoHn

\section{Book Reviews}

A number of copies are still available and may be obtained from the Publishing Manager, British Medical Association, Tavistock Square, W.C.1. price 18s. $6 d$. 